\title{
Comment on Li et al. Non-continuous versus continuous wound drainage after total knee arthroplasty: a meta- analysis
}

\author{
Ruyi Zhang • Jiao Wang $\cdot$ Hua Zhang $\cdot$ Hong Chen • \\ Dehong Cai
}

Received: 11 October 2013 /Accepted: 12 October 2013 / Published online: 6 November 2013

(C) Springer-Verlag Berlin Heidelberg 2013

Dear Editor,

We read with great interest the recent article titled "Noncontinuous versus continuous wound drainage after total knee arthroplasty: a meta- analysis" that was accepted in September 2013 of International Orthopaedics [1]. Li et al. performed a meta-analysis to evaluate non-continuous versus continuous wound drainage after total knee arthroplasty (TKA). It is a valuable study: however, there are some comments we would like to raise related to this article.

1. The electronic databases (Pubmed, Cochrane Library, CNKI, VIP, and WANFANG) were systematically searched by the authors for randomised controlled trials (RCT). Nevertheless, the authors did not focus specifically or in any detail on the issue of the completeness of the search strategy report for databases, which plays an important role in systematic reviews.

2. Manual searches were not clearly stated. The lack of a manual search protocol may be considered a weakness of the meta-analysis.

\footnotetext{
R. Zhang $\cdot$ J. Wang $\cdot$ H. Zhang $\cdot$ H. Chen $\cdot$ D. Cai $(\bowtie)$

Department of Endocrinology, Zhujiang Hospital,

Southern Medical University, 253\# industry road,

510282 Guangzhou, Guangdong, China

e-mail: dehongcaizj@163.com

R. Zhang

e-mail: zhangruyi133@126.com

J. Wang

e-mail: wangjiao150303@163.com

H. Zhang

e-mail: jimzhua@126.com

H. Chen

e-mail: rubychq@163.com
}

3. It is not sufficient that publication bias was only assessed by visual examination of the funnel plot. Funnel plot symmetry should be further assessed by statistical tests (e.g. Egger's linear regression test or Begg's rank correlation test). Moreover, publication bias was only based on haemoglobin loss. In fact, publication bias should be assessed for postoperative visible blood loss, incidence of blood transfusion and complications.

4. There are many kinds of prostheses used in TKA, which might bring different results. If possible, we suggest that a meta-analysis of non-continuous versus continuous wound drainage after TKA with different prostheses be conducted.

5. The authors clearly stated that: "a random-effects model was adopted and further subgroup analysis or sensitivity analysis was performed to explore the potential sources of heterogeneity"; and the pooled analysis of haemoglobin loss between the two groups showed low heterogeneity. However, a sensitivity analysis was conducted in this analysis even though the heterogeneity was low and a fixed-effects model was used.

In conclusion, we agree with the results of this metaanalysis by Li et al. Non-continuous drainage can achieve less haemoglobin loss and postoperative visible blood loss with continuous drainage. Further high-quality RCTs based on larger sample sizes are still needed to confirm the claim.

\section{References}

1. Li T, Zhuang QY, Weng XS, Lei Z (2013) Non-continuous versus continuous wound drainage after total knee arthroplasty: a metaanalysis. Int Orthop. doi:10.1007/s00264-013-2105-0 